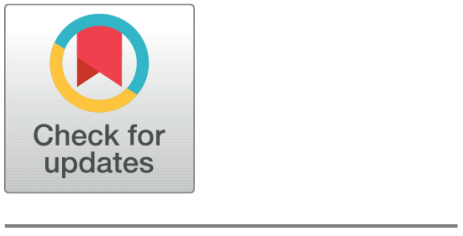

OPEN ACCESS

Received: 15.10 .2020

Accepted: 12.05.2021

Published: 26.05 .2021

Citation: Dubey DK, Rishipathak P (2021) Deciphering Prevalence of Anemia in Reproductive age of Women in Maharashtra, a State of India: A Regional Cross-Sectional Study. Indian Journal of Science and Technology 14(18): 1505-1515. https ://doi.org/10.17485/IJST/v14i18.1867

* Corresponding author.

dubey.dharm@gmail.com

Funding: None

Competing Interests: None

Copyright: (c) 2021 Dubey \& Rishipathak. This is an open access article distributed under the terms of the Creative Commons Attribution License, which permits unrestricted use, distribution, and reproduction in any medium, provided the original author and source are credited.

Published By Indian Society for Education and Environment (iSee)

ISSN

Print: 0974-6846

Electronic: 0974-5645

\section{Deciphering Prevalence of Anemia in Reproductive age of Women in Maharashtra, a State of India: A Regional Cross-Sectional Study}

\author{
Dharmendra Kumar Dubey ${ }^{1 *}$, Parag Rishipathak ${ }^{2}$ \\ 1 Assistant professor, Symbiosis Institute of Health Sciences (SIHS), Symbiosis International \\ (Deemed University), Pune, India \\ 2 Professor \& Director, Symbiosis Centre for Health Skills, Symbiosis International (Deemed \\ University), Pune, India
}

\section{Abstract}

Objectives: India has always been a country with a high prevalence of anemia among women. The objective of the study was to determine the region-specific prevalence of anemia and its determinants among the reproductive age group (15-49 years) of mothers in Maharashtra state. Methods: The fourth round of the national family health survey data was used for this study. It was conducted during 2015-2016 and it provides crucial information on reproductive and child health. It gives national, state and first time at district level of estimates. All women age (15-49) was interviewed during data collection in alternate households in 30 percent selected primary sampling units (PSUs) as per census 2011. For the statistical analysis, multivariable logistic regression models were applied to the data set to assess the trends and social inequalities in terms of anemia through specific regions of Maharashtra. Anemia status is defined by hemoglobin level $(<12 \mathrm{~g} / \mathrm{dl}$ in non-pregnant women, hemoglobin $<11 \mathrm{~g} / \mathrm{dl}$ for pregnant women). Findings: With the help of secondary data analysis, the prevalence of anemia was identified for the Konkan region was 8729 (49.5\%), Paschim Maharashtra was 6465 (48.5\%), Khandesh was 5721 (52.5\%), Marathwada was 4083 (42.7\%) and Vidarbha was 5915 (45.4\%). In Maharashtra, significant regional variation was noticeable in terms of the prevalence of Anemia. The minimum prevalence of anemia was identified in the Marathwada region, which was around $43 \%$ and the maximum prevalence was in the Khandesh region, which was around 53\%. In Maharashtra prevalence of anemia was $48 \%$ out of sample taken from population represented as census 2011. Novelty: This study highlights the prevalence of anemia and risk factors among reproductive age group of women in all five regions of Maharashtra in India.

Keywords: Anemia; Prevalence; Reproductive age; Region; Maharashtra; India 


\section{Introduction}

Maharashtra state distributed in five different regions, each region having dissimilarities in the status of women health. As we know anemia is one of the prevalent health indicators among the reproductive age group of women in Maharashtra, there is a need to explore regional variation among the regions of the state. Anemia is a global public health problem affecting both developing and developed countries at all ages. Women of reproductive age and pregnant women are at a high risk of anemia, which may contribute to maternal morbidity and mortality ${ }^{(1)}$. Anemia is also one of the most common nutritional disorders and it has public health importance in developing countries like India. Where anemia is the most widespread nutritional problem and a common cause of anemia among reproductive age of women. Anemia among pregnant women, leading to premature delivery and low birth weight ${ }^{(2)}$.

In India, the prevalence of anemia reported according to the fourth round of the national family health survey was $53 \%{ }^{(3)}$. Anemia is a condition characterized by a decrease in the concentration of hemoglobin in the blood. Anemia is defined as hemoglobin $(\mathrm{Hb})$ levels $<12.0 \mathrm{~g} / \mathrm{dL}$ in women. Particularly, pregnant women with a hemoglobin level less than $11 \mathrm{~g} / \mathrm{dl}$ considered as anemic, defined by world health organization (WHO). Anemia is a condition in which the number of red blood cells is not sufficient to meet the physiological need of the body ${ }^{(4)}$. Anemia affects an estimated 2.36 billion individuals globally, especially children and women ${ }^{(5)}$. Nutritional status one of the major causes of anemia according to National Health Programmes and prevalence of anemia was varied among the regions ${ }^{(6)}$.

There were multiple common risk factors were contributed to the anemia changes among children and pregnant women in India ${ }^{(7)}$. The prevalence of anemia was found $35.1 \%$ among adolescent females in urban area of Nagpur. Anemia was found with socio-economic status and literacy status of parents ${ }^{(8)}$. In India, anemia was a major cause of the reproductive age of women. It varies across the states. We considered a Maharashtra state to explore the prevalence of Anemia through specific regions. Governments categorized region, geographically, and politically. Not yet, any specific studies performed region-wise in Maharashtra because of the Anemia.

Therefore, we set out to examine the state and regional trends for the prevalence of Anemia and examine how societal discriminations of Anemia among women of reproductive age group have changed over the regions. Because of the existing numerous literature review, there was various discrimination reflected for the prevalence of anemia and its risk factors. In this context, our research questions are like, is there any deciphering prevalence of anemia in the reproductive age group of women among the regions of Maharashtra State. What is the etiology of Anemia among the reproductive age group of women through specific regions of Maharashtra?

In this study, the following objective is as: (ii) to determine the region-specific prevalence of anemia among the reproductive age group of women in Maharashtra State. (ii) to determine the region-specific determinants of anemia in Maharashtra state.

\section{Methods}

\subsection{Data sources}

The International Institute for Population Sciences conducted the fourth round of the national family health survey (NFHS4) under the stewardship of the Ministry of Health and Family Welfare, Government of India. This data nationally, state, and district-level representative and it was conducted during 2015-2016.

\subsection{Sampling Procedure}

NHFS-4 was the first national nutrition survey to be representative at both state and district levels, with data from households. Briefly, these surveys used a two-stage sample design stratified by the urban and rural samples within each state. The first stage involved the selection of primary sampling units, which were villages in rural areas and Census Enumeration Blocks in urban areas. Within each stratum, villages or Census Enumeration Blocks selected from the sampling frame with the probability of selection being proportional to population size. The second stage involved the random selection of 22 households from each primary sampling unit, where a complete household mapping and listing operation conducted before the main survey. Among the selected households, all women aged 15-49 years were eligible for hemoglobin ( $\mathrm{Hb})$ measurement ${ }^{(4)}$.

\subsection{Variable identification and construction}

There are several variables used under this study, which are nominated through outcomes or dependent variable and independent or predictor variables. The outcome of interest was Anemia status. Study variables identified with help of the different type of literature review, which includes the study outcomes and separately described below: 


\subsection{Dependent variables}

Anemia level: Women with any anemia (mild, moderate, severe) are considered for the study. For multivariable logistic regression, the anemia variable is divided into two categories, which are any anemic and not anemic.

\subsection{Independent Variables}

The study includes a set of independent variables to understand the extent and differentials of anemia among women and its effect on the outcomes. The study divides variables into two categories, namely socio-economic \& demographic characteristics/variables. Therefore, numerous independent variables are considered under this study, which is described as a place of living, caste, religion, education, family planning, body mass index, employment, parity, and diet pattern.

\subsection{Statistical Analysis}

Here a multivariable logistic regression model has been developed wherein Anemia category any anemic $=1$ and not anemic $=0$ was used as an outcome variable and (Place of residence), (Caste), (Religion), (Education), (Family planning use), (Body mass index), (Employment), (Parity) and (Diet) were considered as predictor variables.

Equation of the epidemiological model is:

$$
\log \left[\frac{p}{(1-p)}\right]=b_{0}+b_{1} x_{1}+b_{2} x_{2}+b_{3} x_{3}+b_{4} x_{4}+b_{5} x_{5}+b_{6} x_{6}+b_{7} x_{7}+b_{8} x_{8}+b_{9} x_{9}
$$

Where, $b_{1}, b_{2}, b_{3}, b_{4}, \cdots, b_{9}$ are the logistic regression coefficients and $\log \left[\frac{p}{(1-p)}\right]$ is called log odds or logit of the event.

\subsection{Regions in Maharashtra}

The state of Maharashtra includes 35 districts and it was divided into different divisions as well as subdivisions. Maharashtra districts were also divided into five specific regions given geographical, political, and historical significance. Geographically, historically, and according to political sentiments, Maharashtra has five main regions, which are in the following ways: [i] Konkan region (Konkan Division): Mumbai, Mumbai Suburban District, Thane, Raigad, Ratnagiri, and Sindhudurg. [ii] Paschim Maharashtra region (Pune Division): Kolhapur, Pune, Sangli, Satara, and Solapur. [iii] Khandeshregion (Nashik Division): Ahmednagar, Dhule, Jalgaon, Nandurbar, and Nashik. [iv] Marathwada region (Aurangabad Division): Aurangabad, Beed, Hingoli, Jalna, Latur, Nanded, Osmanabad, and Parbhani. [v] Vidarbha region (Nagpur and Amravati divisions): Nagpur division: Bhandara, Chandrapur, Gadchiroli, Gondia, Nagpur and Wardha; Amravati divisions: Akola, Amravati, Buldhana, Yavatmal, and Washim.

\section{Results and Discussion}

Among the adolescent girls, the prevalence of anemia was found to be $55 \%$ in Amaravati city. The contributing factors of anemia were low socioeconomic status and vegetarian diet ${ }^{(9)}$. The regions of Maharashtra, which are known as indifferent appellations: Konkan, Paschim Maharastra, Khandesh, Marathwada, and Vidarbha region. In Maharashtra, the prevalence of Anemia was $48 \%$ and if we see through specific regions then the prevalence of anemia was highest in the Khandesh region and the lowest was in Marathwada (Table 1 ). According to NFHS-3 (2005-2006), prevalence of anemia was $43.7 \%$ in Maharashtra state.

Table 1. Prevalence of anemia according to different regions of Maharashtra through different socio demographic factors

\begin{tabular}{llllll}
\hline & Konkan region & $\begin{array}{l}\text { Paschim Maharashtra } \\
\text { region }\end{array}$ & Khandesh region & $\begin{array}{l}\text { Marathwada } \\
\text { region }\end{array}$ & Vidarbha region \\
\hline Education & & & & & \\
\hline Uneducated & $1289(52.8)$ & $757(47.5)$ & $7869(55.2)$ & $800(38.0)$ & $651(43.4)$ \\
$1-5$ & $1007(52.4)$ & $740(49.6)$ & $2473(53.6)$ & $1918(45.2)$ & $2553(46.9)$ \\
$6-10$ & $3800(51.8)$ & $2929(51.0)$ & $899(52.9)$ & $487(43.3)$ & $1106(44.4)$ \\
$11-12$ & $1211(44.5)$ & $892(45.5)$ & $499(44.2)$ & $293(39.2)$ & $842(43.2)$ \\
$>=13$ & $1421(44.1)$ & $1147(45.0)$ & & & $3006(42.6)$ \\
\hline Religion & & $5420(47.9)$ & $4404(51.0)$ & & Continued on next page \\
\hline Hindu & $6372(48.9)$ & & &
\end{tabular}




\begin{tabular}{|c|c|c|c|c|c|}
\hline \multicolumn{6}{|l|}{ Table 1 continued } \\
\hline Muslim & $1192(48.5)$ & $464(49.2)$ & $1011(59.8)$ & $498(37.6)$ & $560(39.9)$ \\
\hline Christian & $152(49.2)$ & $25(41.0)$ & $29(56.9)$ & $7(53.8)$ & $53(41.4)$ \\
\hline Sikh & $23(29.5)$ & $13(52.0)$ & $0(0.0)$ & $30(63.8)$ & $4(20.0)$ \\
\hline Buddhist & $913(60.4)$ & $464(57.4)$ & $233(51.3)$ & $527(48.0)$ & $814(45.8)$ \\
\hline Other & $77(32.4)$ & $79(43.4)$ & $44(67.7)$ & $17(50.0)$ & $7(17.9)$ \\
\hline \multicolumn{6}{|l|}{ Family Planning use } \\
\hline Not using & $4864(51.1)$ & $3086(47.2)$ & $2821(51.2)$ & $1978(45.1)$ & $2746(46.3)$ \\
\hline $\mathrm{CP}$ & $220(52.4)$ & $59(36.0)$ & $98(48.3)$ & $60(33.5)$ & $54(40.0)$ \\
\hline IUD & $152(61.8)$ & $81(52.6)$ & $135(75.0)$ & $68(50.4)$ & $43(46.2)$ \\
\hline Female Sterilization & $2733(46.7)$ & $2947(50.6)$ & $2332(53.5)$ & $1689(40.4)$ & $2497(44.1)$ \\
\hline Others & $758(47.1)$ & $292(44.9)$ & $336(52.1)$ & $289(42.4)$ & $575(47.6)$ \\
\hline \multicolumn{6}{|l|}{ Caste } \\
\hline SC & $1493(53.6)$ & $1296(50.8)$ & $646(48.8)$ & $900(45.8)$ & $1246(47.7)$ \\
\hline ST & $719(58.6)$ & $460(44.5)$ & $1538(57.9)$ & $359(44.2)$ & $1000(54.4)$ \\
\hline OBC & $2281(50.5)$ & $1249(47.5)$ & $1325(49.6)$ & $811(42.0)$ & $2391(44.3)$ \\
\hline General & $3890(46.5)$ & $3223(48.6)$ & $2076(52.1)$ & $1929(41.2)$ & $1248(40.1)$ \\
\hline \multicolumn{6}{|l|}{ Body Mass Index } \\
\hline Underweight & $2083(60.1)$ & $1428(51.5)$ & $1606(58.7)$ & $1152(46.8)$ & $1824(51.5)$ \\
\hline Normal weight & $4310(49.1)$ & $3393(48.5)$ & $3163(53.0)$ & $2359(43.3)$ & $3290(45.1)$ \\
\hline Overweight & $1643(42.1)$ & $1152(44.5)$ & $710(44.6)$ & $432(34.3)$ & $612(36.2)$ \\
\hline Obese & $693(46.6)$ & $492(50.5)$ & $243(40.5)$ & $142(35.3)$ & $189(37.7)$ \\
\hline \multicolumn{6}{|l|}{ Employment } \\
\hline Not working & $869(44.7)$ & $694(48.1)$ & $478(49.8)$ & $359(43.4)$ & $493(44.8)$ \\
\hline Non-manual & $201(56.0)$ & $129(45.6)$ & $58(59.2)$ & $32(54.2)$ & $34(36.2)$ \\
\hline Agricultural & $49(53.3)$ & $210(49.1)$ & $312(57.8)$ & $240(42.7)$ & $348(51.6)$ \\
\hline Manual & $78(54.9)$ & $32(29.4)$ & $23(60.5)$ & $13(21.0)$ & $17(43.6)$ \\
\hline Total & $1197(47.2)$ & $1065(47.0)$ & $871(53.2)$ & $644(42.6)$ & $892(46.8)$ \\
\hline \multicolumn{6}{|l|}{ Parity } \\
\hline 0 & $3186(51.6)$ & $1747(47.2)$ & 1409 (49.5) & $1036(43.8)$ & $1853(46.9)$ \\
\hline 1 & $1352(44.4)$ & $841(43.0)$ & $802(55.4)$ & $529(46.3)$ & $774(44.6)$ \\
\hline 2 & $2121(48.4)$ & 2257 (50.9) & $1745(53.1)$ & $1071(41.5)$ & $1802(44.7)$ \\
\hline 3 & $1330(54.2)$ & $1157(51.4)$ & $1055(51.5)$ & $878(42.4)$ & $1009(46.6)$ \\
\hline 4 & $509(49.5)$ & $342(47.7)$ & $417(53.4)$ & $385(41.2)$ & $340(42.4)$ \\
\hline $5+$ & $230(41.4)$ & $121(42.8)$ & $293(60.3)$ & $183(39.4)$ & $137(38.8)$ \\
\hline \multicolumn{6}{|l|}{ Diet } \\
\hline Non Vegetarian & $7620(50.1)$ & $5119(48.5)$ & $4192(53.4)$ & $2310(41.5)$ & $4122(45.9)$ \\
\hline Vegetarian & $1109(45.7)$ & $1346(48.5)$ & $1529(50.1)$ & $1773(44.3)$ & $1793(44.3)$ \\
\hline \multicolumn{6}{|l|}{ Place of living } \\
\hline Urban & $6789(48.1)$ & $2930(50.6)$ & $2139(54.3)$ & $1267(45.5)$ & $2070(42.2)$ \\
\hline Rural & $1939(55.3)$ & 3535 (46.9) & $3583(51.5)$ & $2817(41.5)$ & $3844(47.3)$ \\
\hline Total & $8729(49.5)$ & $6465(48.5)$ & $5721(52.5)$ & $4083(42.7)$ & $5915(45.4)$ \\
\hline
\end{tabular}

Table 2 , multivariable logistic regression models have been developed for prediction of anemic mother in comparison to not anemic with help of different predictors for the Konkan region of Maharashtra: Women who were having education 1-5 years were (OR: 1.83 with 95\% CI: 1.29 - 2.60) more likely to be anemic in comparison to uneducated women. Among the higher level of education, probabilities of occurrence of anemia were less likely in comparison to uneducated women. Among the Christian religion were (OR: 2.13 with 95\% CI: 2.29 -3.51) more likely to be anemic in comparison to the Hindu religion of women. As per the National Nutritional Anemia Prophylaxis Program in Mumbai, prevalence of anemia was high among women and it might be control if promote the awareness regarding the National health programs at the community level ${ }^{(10)}$. 
Table 2. Multivariable logistic regression models forprediction of anemic mother than not anemic through different predictors for Konkan

\begin{tabular}{|c|c|c|c|c|}
\hline \multirow{3}{*}{$\begin{array}{l}\text { Predictors } \\
\text { Education }\end{array}$} & \multicolumn{4}{|c|}{ KONKAN REGION } \\
\hline & $\mathrm{P}$ - value & Odds ratio & $95 \% \mathrm{C}$. & \\
\hline & 0.000 & & Lower & Upper \\
\hline Uneducated & - & 1.00 & - & - \\
\hline $1-5$ & 0.00 & 1.83 & 1.29 & 2.60 \\
\hline $6-10$ & 0.75 & 1.05 & 0.78 & 1.40 \\
\hline $11-12$ & 0.10 & 0.73 & 0.50 & 1.07 \\
\hline$>=13$ & 0.10 & 0.74 & 0.52 & 1.06 \\
\hline Religion & 0.006 & & & \\
\hline Hindu & - & 1.00 & - & - \\
\hline Muslim & 0.14 & 0.82 & 0.64 & 1.06 \\
\hline Christian & 0.00 & 2.13 & 1.29 & 3.51 \\
\hline Sikh & 1.00 & 0.00 & 0.00 & 0.00 \\
\hline Buddhist & 0.06 & 1.45 & 0.99 & 2.13 \\
\hline Other & 0.88 & 1.08 & 0.39 & 3.03 \\
\hline Family Planning & 0.195 & & & \\
\hline Not using & - & 1.00 & - & - \\
\hline $\mathrm{CP}$ & 0.29 & 0.68 & 0.33 & 1.39 \\
\hline IUD & 0.78 & 1.12 & 0.51 & 2.44 \\
\hline Female Sterilization & 0.23 & 0.86 & 0.66 & 1.10 \\
\hline Others & 0.18 & 1.24 & 0.90 & 1.72 \\
\hline Caste & 0.000 & & & \\
\hline SC & - & 1.00 & - & - \\
\hline ST & 0.00 & 2.86 & 1.62 & 5.03 \\
\hline OBC & 0.95 & 0.99 & 0.71 & 1.37 \\
\hline General & 0.75 & 1.05 & 0.78 & 1.40 \\
\hline Body Mass Index & 0.000 & & & \\
\hline underweight & - & 1.00 & - & - \\
\hline Normal weight & 0.03 & 0.77 & 0.61 & 0.97 \\
\hline Overweight & 0.00 & 0.54 & 0.41 & 0.72 \\
\hline Obese & 0.00 & 0.54 & 0.37 & 0.79 \\
\hline Employment & 0.020 & & & \\
\hline Not working & - & 1.00 & - & - \\
\hline Non-manual & 0.00 & 1.49 & 1.15 & 1.92 \\
\hline Agricultural & 0.85 & 0.96 & 0.61 & 1.51 \\
\hline Manual & 0.42 & 1.17 & 0.80 & 1.73 \\
\hline Parity & 0.000 & & & \\
\hline 0 & - & 1.00 & - & - \\
\hline 1 & 0.00 & 0.51 & 0.38 & 0.68 \\
\hline 2 & 0.00 & 0.51 & 0.38 & 0.68 \\
\hline 3 & 0.28 & 0.83 & 0.59 & 1.16 \\
\hline 4 & 0.01 & 0.55 & 0.35 & 0.87 \\
\hline $5+$ & 0.62 & 0.87 & 0.50 & 1.52 \\
\hline Diet & 0.000 & & & \\
\hline Non Vegetarian & - & 1.00 & - & - \\
\hline Vegetarian & 0.00 & 1.67 & 1.35 & 2.07 \\
\hline Place & 0.002 & & & \\
\hline Urban & - & 1.00 & - & - \\
\hline Rural & 0.00 & 1.48 & 1.15 & 1.90 \\
\hline
\end{tabular}

Table 3, results of the multivariable logistic regression model were given for the Paschim Maharashtra region: In this region, the prevalence of Anemia was predicted with help of different categories of predictor variables. Women who were having 6-10 years of education and 11-12 years of education were more likely to be anemic in comparison to uneducated women. Religion: Sikh and Buddhists were more likely to be anemic in comparison to uneducated mothers. Family planning: those who were using family planning methods were less likely to be anemic in comparison to no users. 
Table 3. Multivariable logistic regression models for prediction of anemic mother than not anemic through different predictors for Paschim

\begin{tabular}{|c|c|c|c|c|}
\hline & \multicolumn{4}{|c|}{ PASCHIM MAHARASHTRA REGION } \\
\hline & \multirow{2}{*}{$\begin{array}{l}\mathrm{P} \text { - value } \\
0.135\end{array}$} & \multirow[t]{2}{*}{ Odds ratio } & \multicolumn{2}{|c|}{ 95\% Confidence Interval of Odds ratio } \\
\hline Education & & & Lower & Lower \\
\hline Uneducated & - & 1.00 & - & - \\
\hline $1-5$ & 0.72 & 0.94 & 0.66 & 1.33 \\
\hline $6-10$ & 0.32 & 1.16 & 0.87 & 1.55 \\
\hline $11-12$ & 0.14 & 1.32 & 0.92 & 1.91 \\
\hline$>=13$ & 0.79 & 0.95 & 0.67 & 1.35 \\
\hline Religion & 0.217 & & & \\
\hline Hindu & - & 1.00 & - & - \\
\hline Muslim & 0.20 & 0.79 & 0.56 & 1.13 \\
\hline Christian & 1.00 & 0.00 & 0.00 & 0.00 \\
\hline Sikh & 0.11 & 1.48 & 0.92 & 2.38 \\
\hline Buddhist & 0.22 & 1.81 & 0.70 & 4.69 \\
\hline Other & 0.15 & 0.00 & 0.00 & 0.00 \\
\hline Family Planning & 0.150 & & & \\
\hline Not using & - & 1.00 & - & - \\
\hline $\mathrm{CP}$ & 0.01 & 0.29 & 0.11 & 0.77 \\
\hline IUD & 1.00 & 0.00 & 0.00 & 0.00 \\
\hline Female Sterilization & 0.34 & 0.88 & 0.67 & 1.15 \\
\hline Others & 0.39 & 0.84 & 0.57 & 1.24 \\
\hline Caste & 0.002 & & & \\
\hline SC & - & 1.00 & - & - \\
\hline ST & 0.74 & 0.93 & 0.60 & 1.45 \\
\hline OBC & 0.05 & 1.47 & 1.00 & 2.17 \\
\hline General & 0.78 & 0.95 & 0.67 & 1.36 \\
\hline Body Mass Index & 0.003 & & & \\
\hline underweight & - & 1.00 & - & - \\
\hline Normal weight & 0.02 & 0.76 & 0.60 & 0.97 \\
\hline Overweight & 0.00 & 0.61 & 0.46 & 0.82 \\
\hline Obese & 0.92 & 1.02 & 0.70 & 1.47 \\
\hline Employment & 0.015 & & & \\
\hline Not working & - & 1.00 & - & - \\
\hline Non-manual & 0.26 & 0.85 & 0.65 & 1.13 \\
\hline Agricultural & 0.33 & 1.14 & 0.88 & 1.48 \\
\hline Manual & 0.01 & 0.53 & 0.34 & 0.82 \\
\hline Parity & 0.083 & & & \\
\hline 0 & - & 1.00 & - & - \\
\hline 1 & 0.02 & 0.71 & 0.53 & 0.94 \\
\hline 2 & 0.67 & 0.94 & 0.69 & 1.27 \\
\hline 3 & 0.77 & 0.94 & 0.64 & 1.39 \\
\hline 4 & 0.90 & 1.04 & 0.60 & 1.77 \\
\hline $5+$ & 0.18 & 1.60 & 0.81 & 3.17 \\
\hline Diet & 0.091 & & & \\
\hline Non Vegetarian & - & 1.00 & - & - \\
\hline Vegetarian & 0.09 & 0.83 & 0.67 & 1.03 \\
\hline Place & 0.008 & & & \\
\hline Urban & - & 1.00 & - & - \\
\hline Rural & 0.01 & 0.76 & 0.62 & 0.93 \\
\hline
\end{tabular}

Table 4, findings of the Khandesh region was presented to predict the prevalence of anemia among the anemic women in comparison to not anemic women through individual explanatory variables. Probability values and odds ratio with $95 \%$ confidence interval was given for specific categories of explanatory variables. Family planning: Among the female sterilization, the prevalence of Anemia is higher in comparison to no users. Caste: Among Schedule tribe and OBC were more likely to be anemic in comparison to schedule caste. Body mass index: Among the normal, overweight, and obese less likely to be anemic in comparison to underweight women. 
Table 4. Multivariable logistic regression models for prediction of anemic mother than not anemic through different predictors for Khandesh Region of Maharashtra.

\begin{tabular}{|c|c|c|c|c|}
\hline & \multicolumn{4}{|c|}{ KHANDESH REGION } \\
\hline & $\mathrm{P}$ - value & Odds ratio & $95 \% \mathrm{Cc}$ & r Odds ratio \\
\hline Education & 0.398 & & Lower & Upper \\
\hline Uneducated & - & 1.00 & - & - \\
\hline $1-5$ & 0.16 & 1.33 & 0.89 & 2.00 \\
\hline $6-10$ & 0.36 & 1.16 & 0.84 & 1.59 \\
\hline $11-12$ & 0.09 & 1.41 & 0.94 & 2.11 \\
\hline$>=13$ & 0.13 & 1.43 & 0.90 & 2.26 \\
\hline Religion & 0.424 & & & \\
\hline Hindu & - & 1.00 & - & - \\
\hline Muslim & 0.04 & 1.58 & 1.02 & 2.45 \\
\hline Christian & 0.45 & 1.42 & 0.57 & 3.54 \\
\hline Sikh & 1.00 & 0.00 & 0.00 & 0.00 \\
\hline Buddhist & 0.45 & 1.29 & 0.66 & 2.53 \\
\hline Other & 0.57 & 1.47 & 0.39 & 5.52 \\
\hline Family Planning & 0.017 & & & \\
\hline Not using & - & 1.00 & - & - \\
\hline $\mathrm{CP}$ & 0.27 & 0.70 & 0.37 & 1.32 \\
\hline IUD & 0.01 & 0.17 & 0.05 & 0.63 \\
\hline Female Sterilization & 0.35 & 1.16 & 0.85 & 1.58 \\
\hline Others & 0.23 & 0.76 & 0.48 & 1.19 \\
\hline Caste & 0.000 & & & \\
\hline SC & - & 1.00 & - & - \\
\hline ST & 0.44 & 1.22 & 0.74 & 1.99 \\
\hline OBC & 0.66 & 1.11 & 0.68 & 1.81 \\
\hline General & 0.29 & 0.77 & 0.48 & 1.24 \\
\hline Body Mass Index & 0.010 & & & \\
\hline Underweight & - & 1.00 & - & - \\
\hline Normal weight & 0.01 & 0.70 & 0.54 & 0.92 \\
\hline Overweight & 0.01 & 0.63 & 0.43 & 0.91 \\
\hline Obese & 0.01 & 0.46 & 0.27 & 0.80 \\
\hline Employment & 0.037 & & & \\
\hline Not working & - & 1.00 & - & - \\
\hline Non-manual & 0.03 & 1.63 & 1.04 & 2.56 \\
\hline Agricultural & 0.06 & 1.29 & 0.99 & 1.69 \\
\hline Manual & 0.11 & 1.78 & 0.88 & 3.60 \\
\hline Parity & 0.003 & & & \\
\hline 0 & - & 1.00 & - & - \\
\hline 1 & 0.00 & 1.98 & 1.35 & 2.89 \\
\hline 2 & 0.03 & 1.49 & 1.05 & 2.13 \\
\hline 3 & 0.80 & 1.05 & 0.70 & 1.58 \\
\hline 4 & 0.22 & 1.44 & 0.81 & 2.56 \\
\hline $5+$ & 0.14 & 1.60 & 0.85 & 3.00 \\
\hline Diet & 0.253 & & & \\
\hline Non Vegetarian & - & 1.00 & - & - \\
\hline Vegetarian & 0.25 & 0.87 & 0.69 & 1.10 \\
\hline Place & 0.005 & & & \\
\hline Urban & - & 1.00 & - & - \\
\hline Rural & 0.01 & 1.47 & 1.12 & 1.93 \\
\hline
\end{tabular}

Table 5, presented for the Marathwada region to predict the prevalence of anemia in comparison to not anemic women. Probability value and odds ratio with 95\% confidence interval was given for specific categories of the predictors. Among these predictors like categories of family planning, body mass index, employment, and places were more or less likely to be anemic with its counterpart. 
Table 5. Multivariable logistic regression models for prediction of anemic mother than not anemic through different predictors for MARATHWADA REGION of Maharashtra

\begin{tabular}{|c|c|c|c|c|}
\hline & \multicolumn{2}{|c|}{ MARATHWADA REGION } & \multirow{2}{*}{\multicolumn{2}{|c|}{ 95\% Confidence Interval for Odds ratio }} \\
\hline & $\mathrm{P}$ - value & Odds ratio & & \\
\hline Education & 0.183 & & Lower & Upper \\
\hline Uneducated & - & 1.00 & - & - \\
\hline $1-5$ & 0.03 & 1.49 & 1.04 & 2.12 \\
\hline $6-10$ & 0.47 & 1.12 & 0.82 & 1.55 \\
\hline $11-12$ & 0.81 & 1.06 & 0.68 & 1.65 \\
\hline$>=13$ & 0.76 & 0.92 & 0.52 & 1.62 \\
\hline Religion & 0.776 & & & \\
\hline Hindu & - & 1.00 & - & - \\
\hline Muslim & 0.79 & 0.95 & 0.66 & 1.37 \\
\hline Christian & 0.93 & 1.13 & 0.07 & 17.06 \\
\hline Sikh & 0.19 & 0.75 & 0.49 & 1.15 \\
\hline Buddhist & 0.99 & 0.99 & 0.18 & 5.38 \\
\hline Other & 0.00 & 0.00 & 0.00 & 0.00 \\
\hline Family Planning & 0.037 & & & \\
\hline Not using & - & 1.00 & - & - \\
\hline $\mathrm{CP}$ & 0.74 & 0.86 & 0.36 & 2.08 \\
\hline IUD & 0.19 & 1.90 & 0.73 & 4.96 \\
\hline Female Sterilization & 0.22 & 0.81 & 0.58 & 1.13 \\
\hline Others & 0.01 & 0.55 & 0.36 & 0.86 \\
\hline Caste & 0.801 & & & \\
\hline $\mathrm{SC}$ & - & 1.00 & - & - \\
\hline ST & 0.29 & 0.76 & 0.45 & 1.27 \\
\hline OBC & 0.81 & 0.95 & 0.63 & 1.44 \\
\hline General & 0.41 & 0.86 & 0.59 & 1.24 \\
\hline Body Mass Index & 0.000 & & & \\
\hline Underweight & - & 1.00 & - & - \\
\hline Normal weight & 0.35 & 0.89 & 0.69 & 1.14 \\
\hline Overweight & 0.00 & 0.47 & 0.33 & 0.68 \\
\hline Obese & 0.00 & 0.32 & 0.14 & 0.70 \\
\hline Employment & 0.002 & & & \\
\hline Not working & - & 1.00 & - & - \\
\hline Non-manual & 0.11 & 1.60 & 0.91 & 2.84 \\
\hline Agricultural & 0.75 & 1.04 & 0.80 & 1.36 \\
\hline Manual & 0.00 & 0.32 & 0.17 & 0.62 \\
\hline Parity & 0.240 & & & \\
\hline 0 & - & 1.00 & - & - \\
\hline 1 & 0.15 & 1.33 & 0.90 & 1.97 \\
\hline 2 & 0.05 & 1.45 & 1.00 & 2.12 \\
\hline 3 & 0.15 & 1.38 & 0.89 & 2.15 \\
\hline 4 & 0.43 & 1.23 & 0.74 & 2.06 \\
\hline $5+$ & 0.72 & 0.89 & 0.48 & 1.66 \\
\hline Diet & 0.438 & & & \\
\hline Non Vegetarian & - & 1.00 & - & - \\
\hline Vegetarian & 0.44 & 0.90 & 0.70 & 1.17 \\
\hline Place & 0.001 & & & \\
\hline Urban & - & 1.00 & - & - \\
\hline Rural & 0.00 & 0.62 & 0.47 & 0.82 \\
\hline
\end{tabular}

Table 6, represents the Vidarbha region to predict the prevalence of anemia through the predictors. Probability value and odds ratio with $95 \%$ confidence interval was given for each category of individual predictors. 
Table 6. Multivariable logistic regression models for prediction of anemic mother than not anemic through different predictors for Vidarbha Region of Maharashtra.

\begin{tabular}{|c|c|c|c|c|}
\hline & \multicolumn{4}{|c|}{ VIDARBHA REGION } \\
\hline & $\mathrm{P}$ - value & Odds ratio & $95 \% \mathrm{C}$ & Odds ratio \\
\hline Education & 0.034 & & Lower & Upper \\
\hline Uneducated & - & 1.00 & - & - \\
\hline $1-5$ & 0.11 & 1.39 & 0.93 & 2.08 \\
\hline $6-10$ & 0.21 & 1.25 & 0.88 & 1.76 \\
\hline $11-12$ & 0.01 & 1.67 & 1.12 & 2.49 \\
\hline$>=13$ & 0.01 & 1.79 & 1.14 & 2.80 \\
\hline Religion & 0.034 & & & \\
\hline Hindu & - & 1.00 & - & - \\
\hline Muslim & 0.46 & 1.18 & 0.76 & 1.84 \\
\hline Christian & 0.02 & 0.44 & 0.22 & 0.89 \\
\hline Sikh & 0.43 & 0.44 & 0.06 & 3.40 \\
\hline Buddhist & 0.07 & 0.66 & 0.43 & 1.03 \\
\hline Other & 0.11 & 0.22 & 0.03 & 1.39 \\
\hline Family Planning & 0.013 & & & \\
\hline Not using & - & 1.00 & - & - \\
\hline $\mathrm{CP}$ & 0.07 & 0.39 & 0.14 & 1.08 \\
\hline IUD & 0.26 & 1.88 & 0.63 & 5.64 \\
\hline Female Sterilization & 0.01 & 0.65 & 0.47 & 0.91 \\
\hline Others & 0.86 & 1.04 & 0.70 & 1.54 \\
\hline Caste & 0.014 & & & \\
\hline SC & - & 1.00 & - & - \\
\hline ST & 0.21 & 0.75 & 0.48 & 1.18 \\
\hline OBC & 0.00 & 0.56 & 0.38 & 0.83 \\
\hline General & 0.00 & 0.54 & 0.35 & 0.82 \\
\hline Body Mass Index & 0.00 & & & \\
\hline Underweight & - & 1.00 & - & - \\
\hline Normal weight & 0.33 & 0.90 & 0.72 & 1.12 \\
\hline Overweight & 0.00 & 0.57 & 0.41 & 0.80 \\
\hline Obese & 0.00 & 0.23 & 0.12 & 0.45 \\
\hline Employment & 0.020 & & & \\
\hline Not working & - & 1.00 & - & - \\
\hline Non-manual & 0.14 & 0.70 & 0.44 & 1.12 \\
\hline Agricultural & 0.01 & 1.37 & 1.08 & 1.74 \\
\hline Manual & 0.69 & 0.87 & 0.45 & 1.70 \\
\hline Parity & 0.193 & & & \\
\hline 0 & - & 1.00 & - & - \\
\hline 1 & 0.26 & 1.22 & 0.86 & 1.73 \\
\hline 2 & 0.24 & 1.25 & 0.86 & 1.79 \\
\hline 3 & 0.02 & 1.69 & 1.11 & 2.58 \\
\hline 4 & 0.40 & 1.27 & 0.73 & 2.20 \\
\hline $5+$ & 0.71 & 1.14 & 0.57 & 2.30 \\
\hline Diet & 0.200 & & & \\
\hline Non Vegetarian & - & 1.00 & - & - \\
\hline Vegetarian & 0.20 & 0.87 & 0.70 & 1.08 \\
\hline Place & 0.627 & & & \\
\hline Urban & - & 1.00 & - & - \\
\hline Rural & 0.63 & 1.06 & 0.84 & 1.34 \\
\hline
\end{tabular}

Most previous studies have been done and demonstrated the optimal translation of experience and knowledge for desired overall improvement in health ${ }^{(11)}$. The present study concluded the prevalence of Anemia with exclusively all regions of Maharashtra. The regions were Konkan, Paschim Maharastra, Khandesh, Marathwada, and Vidarbha, among the region's prevalence of Anemia estimated according to various predictor variables. This information may serve as a benchmark to achieve the sustainable development Goals (SDG) by 2030. Which India committed too. Prevalence of anemia among pregnant women in India gradually declined over the year ${ }^{(12)}$. Anemia control program is more resourceful to control the prevalence of anemia 
and the awareness of the risk factors to prevent the anemia ${ }^{(13)}$. It is important to aware about key risk factors and implementing interventions for controlling the anemia in selected population ${ }^{(14,15)}$. The prevalence of anemia was very high and it shows that anemia is a major public health problem, so government needs to take the extra efforts to promote the health of an individual, community as well as the country ${ }^{(16)}$. The prevalence of anemia in India was 53\% among women of reproductive age (15-49) as of NFHS-4 (2015-2016) and highest value over the past 26 years was 55.40 in 1990 and lowest was 51.10 in $2014{ }^{(17)}$.

\section{Conclusion}

In Maharashtra State, the prevalence of Anemia was $48 \%$ and the highest prevalence of anemia was in the Khandesh region (Identified risk factors: uneducated, others religion, IUD family planning users, schedule tribe caste, underweight, manual working, fifth and above parity, non-vegetarian and urban place). Respectively, the prevalence of anemia was in the Kokan region (Identified risk factors: uneducated, Buddhist religion, IUD family planning users, schedule tribe caste, underweight, non-manual working, third parity, non-vegetarian and rural place). Respectively, the prevalence of anemia was in the Paschim Maharashtra region (Identified risk factors: 6-10 years education, Buddhist religion, IUD family planning users, schedule caste, underweight, agriculture working, third parity, non-vegetarian and urban place). Respectively, the prevalence of anemia was in the Marathwada region (Identified risk factors: 6-10 years education, Sikh religion, IUD family planning users, schedule caste, underweight, non-manual working, first parity, vegetarian, and urban place). Respectively, the prevalence of anemia was in the Vidarbha region (Identified risk factors: 6-10 years education, Hindu religion, other family planning users, schedule tribe caste, underweight, agriculture working, third parity, non-vegetarian and rural place). Across the region, underweight was a common risk factors of anemia.

\section{Limitations}

The present study was limited to the reproductive age group of a mother (15-49) and few regions of Maharashtra state.

\section{Acknowledgement}

We thank to entire team of the NFHS-4, who was involved during the process of data collection.

\section{References}

1) Gautam S, Min H, Kim H, Jeong HS. Determining factors for the prevalence of anemia in women of reproductive age in Nepal: Evidence from recent national survey data. PLoS ONE. 2019;14(6):e0218288. Available from: https://doi.org/10.1371/journal.pone.0218288.

2) Dubey DK, Nath DC. An Epidemiological Model Investigating the Association between Mothers Nutritional Status and Low Birth Weight in India. Health. 2016;08(03):251-261. Available from: https://dx.doi.org/10.4236/health.2016.83027.

3) Dubey DK, Rishipathak P, Das A. Varying Patterns of Societal Discriminations in Anaemia among Women in India: Findings from Third and Fourth rounds of NFHS Survey. Indian Journal of Public Health Research \& Development. 2019;10(5):759-759. Available from: https://dx.doi.org/10.5958/09765506.2019.01103.3.

4) International Institute for Population Sciences (IIPS) and ICF. National Family Health Survey (NFHS-4). 2015-16. Available from: http://rchiips.org/nfhs/ nfhs-4Reports/India.pdf.

5) Vos T, Allen C, Arora M, Barber RM, qar A Bhutta Z, Brown A, et al. Global, regional, and national incidence, prevalence, and years lived with disability for 310 diseases and injuries, 1990- 2015: a systematic analysis for the Global Burden of Disease Study. The Lancet. 2016;388(10053):1545-1602. Available from: https://doi.org/10.1016/S0140-6736(16)31678-6.

6) Srivastava R, Dudeja P, Bhide G, Pant M. Demystifying prevalence of anemia in female spouses of army personnel in an Army's Corps zone: A multicentric cross-sectional study. International Journal of Medical Science and Public Health. 2019;8(5):365-368. Available from: https://dx.doi.org/10.5455/ijmsph. 2019.0203414032019.

7) Nguyen P, Scott S, Avula R, Tran L, Menon P. Trends and drivers of change in the prevalence of Anemia among 1 million women and children in India. BMJ Glob Health. 2006;3. Available from: http://dx.doi.org/10.1136/bmjgh-2018-001010.

8) Chaudhary S, Dhage V. A study of anemia among adolescent females in the urban area of Nagpur. Indian Journal of Community Medicine. 2008;33(4):243243. Available from: https://dx.doi.org/10.4103/0970-0218.43230.

9) Jawarkar A, Lokare P, Kizhatil A, Jawarkar J. Prevalence of anemia and effectiveness of iron supplementation in anemic adolescent school girls at Amravati City (Maharashtra). Journal of Health Research and Reviews. 2015;2:7-10. Available from: https://dx.doi.org/10.4103/2394-2010.158122.

10) Shidhaye PR, Nagaonkar SN, Giri PA, Shidhaye RR. Prevalence of anemia in the postnatal women at a tertiary care teaching hospital in Mumbai. Journal of Medical Nutrition and Nutraceuticals. 2012;1:54-57. Available from: https://dx.doi.org/10.4103/2278-019x.94630.

11) Dubey DK, Das A. Socio-Demographic Determinants of Anemia among Women: A Study about Empowered Action Group (EAG) States of India. Indian Journal of Public Health Research \& Development. 2019;10(5):720-723. Available from: https://dx.doi.org/10.5958/0976-5506.2019.01096.9.

12) .. Available from: https://data.worldbank.org/indicator/SH.PRG.ANEM?locations=IN.

13) Bansal R, Bedi M, Kaur J, Kaur K, Shergill HK, Khaira HK, et al. Prevalence and factors associated with anemia among pregnant women attending antenatal clinic. Adesh University Journal of Medical Sciences \& Research. 2020;2(1):42-48. Available from: https://dx.doi.org/10.25259/aujmsr_8_2020.

14) Nath A, Vindhya J, Murthy GS, Metgud C, Sheeba B, Shubhashree V, et al. Prevalence and risk factors of anemia among pregnant women attending a public-sector hospital in Bangalore, South India. Journal of Family Medicine and Primary Care. 2019;8(1):37-43. Available from: https://dx.doi.org/10. 
4103/jfmpc.jfmpc_265_18.

15) Mahamoud NK, Mwambi B, Oyet C, Segujja F, Webbo F, Okiria JC, et al. $<\mathrm{p}>$ Prevalence of Anemia and Its Associated Socio-Demographic Factors Among Pregnant Women Attending an Antenatal Care Clinic at Kisugu Health Center IV, Makindye Division, Kampala, Uganda $</ \mathrm{p}>$. Journal of Blood Medicine. 2020;11:13-18. Available from: https://dx.doi.org/10.2147/jbm.s231262.

16) Singh M, Kishore S, Jain B, Verma N, Gawande K, Kishore S. A study to assess prevalence of anaemia among beneficiaries of Anaemia Mukt Bharat Campaign in Uttarakhand. Journal of Family Medicine and Primary Care. 2020;9(3):1691-1694. Available from: https://dx.doi.org/10.4103/jfmpc.jfmpc 941_19.

17) .. Available from: https://www.indexmundi.com/facts/india/prevalence-of-anemia. 